\title{
The error function in the study of singularly perturbed convection-diffusion problems with discontinuous boundary data
}

\author{
J.L. López ${ }^{1}$, E. Pérez Sinusía ${ }^{1}$ and N.M. Temme ${ }^{2}$ \\ 1 Dpto. de Ingeniería Matemática e Informática, Universidad Pública de Navarra, \\ Pamplona 31006, Spain \\ 2 CWI, PO Box 94079, 1090 GB Amsterdam, The Netherlands
}

Summary. We show the importance of the error function in the approximation of the solution of singularly perturbed convection-diffusion problems with discontinuous boundary conditions. It is observed that the error function (or a combination of them) provides an excellent approximation and reproduces accurately the effect of the discontinuities on the behaviour of the solution at the boundary and interior layers.

\section{Introduction}

We consider the model convection-diffusion problem $-\varepsilon \Delta U+\vec{v} \cdot \vec{\nabla} U=0$ in $\Omega$ where $\Omega$ is an open set in $\mathbb{R}^{2}$ or $\mathbb{R}^{3}, \varepsilon>0$ and $\vec{v}$ is a constant vector. Besides the small perturbation parameter $\varepsilon$, other sources of singular behaviour for the solution of singular perturbation problems are the discontinuities of the boundary data. We consider for this problem Dirichlet boundary data piecewise constant: $\left.U\right|_{\partial \Omega}=0$ or 1 with jump discontinuities (of height 1) at some points in $\partial \Omega$.

In $[1,2,3,4,5,6,7]$, we have analyzed this problem in a number of two and three-dimensional unbounded and bounded domains $\Omega$ with discontinuous boundary data at $\partial \Omega$. For all these problems, we have found that the solution in the singular limit $\varepsilon \rightarrow 0^{+}$and away from the discontinuity points of the boundary data can be approximated in the form

$$
U=U_{0}(1+\mathcal{O}(\sqrt{\varepsilon}))
$$

where $U_{0}$ is an error function or a combination of error functions. In the next section we describe the asymptotic approximation and layer structure of the solutions found in examples considered in our earlier papers. In the conclusion section we discuss the (in our opinion) universality of the complementary error function as basic approximant of the solution of this kind of problems. 
In what follows, we will consider the polar coordinates $x=r \sin \phi, y=$ $r \cos \phi, \vec{r}:=(x, y), w:=1 /(2 \varepsilon)$ and $\zeta(x, y):=\sqrt{r-x \sin \beta-y \cos \beta}$. In all the problems analyzed we consider $U \in \mathcal{C}(\tilde{\Omega}) \cap \mathcal{D}^{2}(\Omega)$ and $U$ bounded on bounded subsets of $\tilde{\Omega}$, where $\tilde{\Omega}$ is the closed set $\bar{\Omega}$ indented at the discontinuity points of the boundary conditions.

\section{Examples in two-dimensional domains}

In this section we consider $\vec{v}=(\sin \beta, \cos \beta)$, with $\beta \in[0, \pi / 2)$.

\subsection{A quarter plane}

For $(x, y) \in \tilde{\Omega}_{1}:=\bar{\Omega}_{1} \backslash\{(0,0)\}$ and $0 \leq \beta<\pi / 2$ the solution of the problem

$$
\left\{\begin{array}{l}
-\varepsilon \Delta U+\vec{v} \cdot \vec{\nabla} U=0, \quad(x, y) \in \Omega_{1}:=(0, \infty) \times(0, \infty), \\
U(x, 0)=0, \quad U(0, y)=1,
\end{array}\right.
$$

can be approximated in $\tilde{\Omega}_{1}$ by (1) with $U_{0}(x, y)=\operatorname{erfc}[\sqrt{w \zeta(x, y)}]$ for $\beta=0$ and

$$
U_{0}(x, y)=\frac{1}{2} \operatorname{erfc}[\sqrt{w \zeta(x, y)}], \quad \text { for } 0<\beta<\pi / 2 .
$$

Then, the first order approximation is a complementary error function that
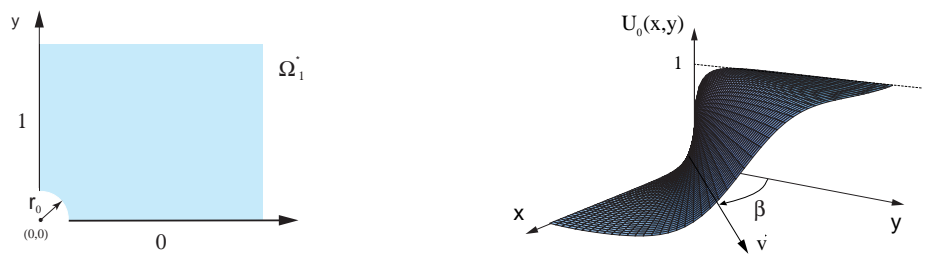

Fig. 1. (a) Indented region $\tilde{\Omega}_{1}$ (b) First order approximation $U_{\pi / 4}^{0}(x, y)$ to the solution of $\left(P_{1}\right)$ for $\varepsilon=0.1$ and $\beta=\pi / 4$. Near the half-line $t \vec{v}, t>0$ an internal parabolic layer occurs.

exhibits an interior layer of width $\mathcal{O}(\sqrt{\varepsilon})$ and parabolic level lines of equation $r-\vec{v} \cdot \vec{r}=C \cdot \varepsilon$ near the half-line $t \vec{v}, t>0$ (see Figure 1 (b)). For further details we refer to [1].

\subsection{An infinite strip}

For $(x, y) \in \tilde{\Omega}_{2}:=\bar{\Omega}_{2} \backslash\{(a, 0),(b, 0)\}$ and $0 \leq \beta \leq \pi / 2$, the solution of

$$
\begin{cases}-\varepsilon \Delta U+\vec{v} \cdot \vec{\nabla} U=0, & (x, y) \in \Omega_{2}:=(-\infty, \infty) \times(0,1), \\ U(x, 0)=\chi_{[a, b]}(x), U(x, 1)=0, & a<b,\end{cases}
$$


in $\tilde{\Omega}_{2}$ is of the form (1) with

$$
\begin{aligned}
U_{0}(x, y)= & \frac{1+\delta_{\beta, \pi / 2}}{2}\left\{\operatorname{sign}\left[\beta-\arctan \left(\frac{x-a}{y}\right)\right] \operatorname{erfc}(\sqrt{w \zeta(x-a, y)})\right. \\
& -e^{2(y-1) w \cos \beta} \operatorname{sign}\left[\beta-\arctan \left(\frac{x-a}{2-y}\right)\right] \operatorname{erfc}(\sqrt{w \zeta(x-a, 2-y)}) \\
& -\operatorname{sign}\left[\beta-\arctan \left(\frac{x-b}{y}\right)\right] \operatorname{erfc}(\sqrt{w \zeta(x-b, y)}) \\
& \left.+e^{2(y-1) w \cos \beta} \operatorname{sign}\left[\beta-\arctan \left(\frac{x-b}{2-y}\right)\right] \operatorname{erfc}(\sqrt{w \zeta(x-b, 2-y)})\right\} \\
& +\frac{1}{2}\left[\chi_{A}(x, y)+\chi_{A_{0}}(x, y)-e^{2(y-1) w \cos \beta}\left(\chi_{B}(x, y)+\chi_{B_{0}}(x, y)\right)\right] .
\end{aligned}
$$

The region $A$ is limited by the lines $y=0, y=1, x=a+y \tan \beta$ and $x=$ $b+y \tan \beta$. Region $B$ is limited by the lines $y=0, y=1, x=a+(2-y) \tan \beta$ and $x=b+(2-y) \tan \beta$.
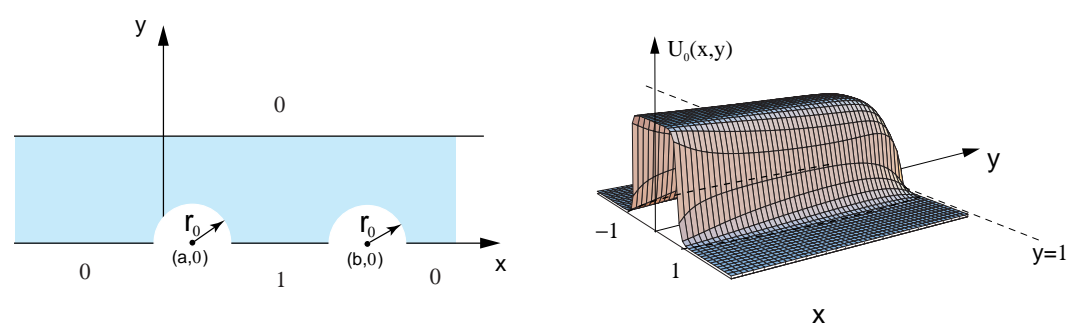

Fig. 2. (a) Indented region $\tilde{\Omega}_{2}$ (b) Graph of the first order approximation, $U_{\beta}^{0}(x, y)$, to the solution of the problem $\left(P_{2}\right)$ for $\varepsilon=0.1$ and $\beta=0$.

In this case, $U_{\beta}^{0}$ is a combination of four error functions plus step functions multiplied by exponential functions of $y$, and it exhibits two interior layers of width $\mathcal{O}(\sqrt{\varepsilon})$ and level lines of equation $\zeta(x-c, y)=$ constant with $c=a, b$. It presents a regular boundary layer of width $\mathcal{O}(\varepsilon)$ near the piece of the outflow boundary situated between the points $(a+\tan \beta, 1)$ and $(b+\tan \beta, 1)$ and it also exhibits two corner layers of area $\mathcal{O}(\sqrt{\varepsilon}) \times \mathcal{O}(\varepsilon)$ near the points $(a+\tan \beta, 1)$ and $(b+\tan \beta, 1)$ (see Figure $2(\mathrm{~b}))$. The reader is referred to [1] for further information.

\subsection{A rectangle}

For $(x, y) \in \tilde{\Omega}_{3}:=\bar{\Omega}_{3} \backslash\{(0,0),(\pi a, 0)\}$ and $\beta \in(0, \pi / 2]$, the solution of

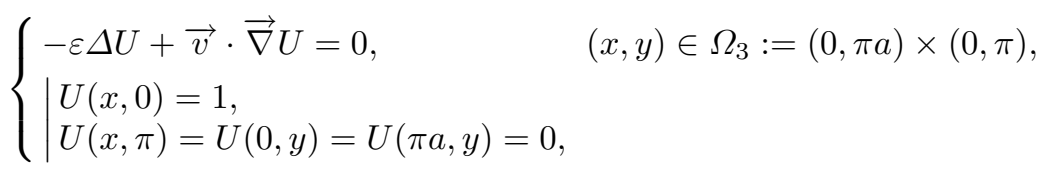


where $0 \leq \beta<2 \pi$ and $a>0$, can be approximated in $\tilde{\Omega}_{3}$ by (1) with

$$
\begin{aligned}
U_{0}(x, y)= & e^{w y \cos \beta} \frac{\sinh [(\pi-y) w \cos \beta]}{\sinh [\pi w \cos \beta]} \times\left\{\chi_{A}(x, y)-e^{2 w(x-\pi a) \sin \beta} \chi_{B}(x, y)\right. \\
& +\frac{\left(1+\delta_{\beta, \pi / 2}\right)}{2}\left[\operatorname{sign}\left(\beta-\arctan \left(\frac{x}{y}\right)\right) \operatorname{erfc} \sqrt{w \zeta(x, y)}\right. \\
& -e^{2(x-\pi a) w \sin \beta} \operatorname{sign}\left(\beta-\arctan \left(\frac{2 \pi a-x}{y}\right)\right) \operatorname{erfc} \sqrt{w \zeta(2 \pi a-x, y)} \\
& \left.\left.+e^{2(x-\pi a) w \sin \beta} \operatorname{sign}\left(\beta-\arctan \left(\frac{\pi a-x}{y}\right)\right) \operatorname{erfc} \sqrt{w \zeta(\pi a-x, y)}\right]\right\} .
\end{aligned}
$$

The regions $A$ and $B$ are defined by $A:=\left\{(x, y) \in \Omega_{3}, y<x \cot \beta\right\}$ and $B:=$ $\left\{(x, y) \in \Omega_{3},(\pi a-x) \cot \beta<y<(2 \pi a-x) \cot \beta\right\}$.

Then, the first order approximation of the solution of $\left(P_{3}\right)$ is a linear combination of error functions and elementary functions. The error functions present interior/boundary layers of width $\mathcal{O}(\sqrt{\varepsilon})$. The exponential factors are giving boundary layers of width $\mathcal{O}(\varepsilon)$ (see Figure $3(\mathrm{~b})$ ). For more details we refer to [5].
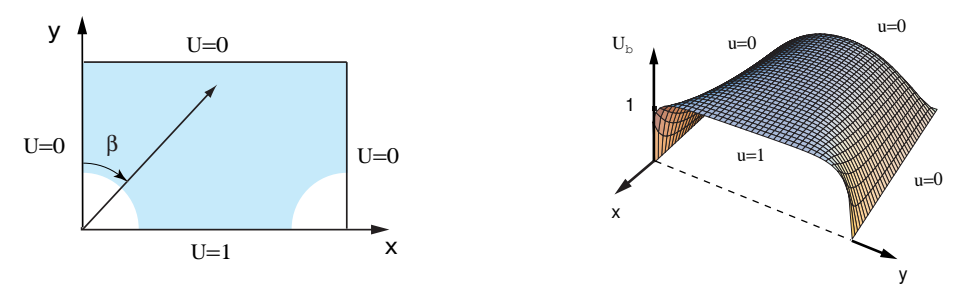

Fig. 3. (a) Indented region $\tilde{\Omega}_{3}$ (b) First order approximation $U_{\beta}^{0}(x, y)$ to problem $\left(P_{3}\right)$ for $\varepsilon=0.1$ and $\beta=0$.

\section{Examples in three-dimensional domains}

\subsection{An octant}

For $(x, y, z) \in \tilde{\Omega}_{4}:=\Omega_{4} \cup\{(x, y, 0) ; x, y>0\} \cup\{(0, y, z) ; y \geq 0, z>0\} \cup$ $\{(x, 0, z) ; x \geq 0, z>0\}$ (see Figure 4 (a)), the solution of the problem

$\begin{cases}-\varepsilon \Delta U+U_{z}=0, & \text { in } \Omega_{4}:=(0, \infty) \times(0, \infty) \times(0, \infty), \\ U(x, y, 0)=1, U(0, y, z)=U(x, 0, z)=0, & \text { for }(x, y, z) \in \tilde{\Omega}_{4},\end{cases}$

can be approximated in $\tilde{\Omega}_{4}$ by (1) with 

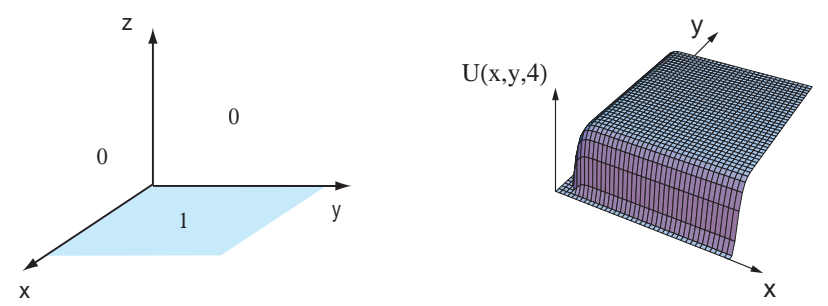

Fig. 4. (a) Domain $\Omega_{4}$ and Dirichlet conditions of problem $\left(P_{4}\right)$ (b) Graph of the first order approximation for the solution of problem $\left(P_{4}\right)$ for $\varepsilon=0.1$.

$$
U_{0}(x, y, z)=\operatorname{erf} \sqrt{w \zeta(x, z)} \operatorname{erf} \sqrt{w \zeta(y, z)}
$$

The solution of this problem has boundary layers along the planes $x=0$ and $y=0$ of size $\mathcal{O}(\sqrt{\varepsilon})$ (see Figure 4 (b)). For further information consult $[4,6]$.

\subsection{A cuboid}

For $(x, y, z) \in \widetilde{\Omega}_{5}:=\bar{\Omega}_{5} \backslash\{\{(0, y, 0),(\pi a, y, 0) ; 0 \leq y \leq \pi b\} \cup\{(x, 0,0),(x, \pi b, 0) ; 0 \leq x \leq \pi a\}\}$ (see Figure 5 (a)), the solution of the problem

$\begin{cases}-\varepsilon \Delta U+U_{z}=0 & \text { in } \Omega_{5}:=(0, \pi a) \times(0, \pi b) \times(0, \pi), \\ U(0, y, z)=U(\pi a, y, z)=U(x, 0, z)=0, & \text { for }(x, y, z) \in \widetilde{\Omega}_{5} . \\ U(x, \pi b, z)=U(x, y, \pi)=0, U(x, y, 0)=1, & \end{cases}$

can be approximated in $\tilde{\Omega}_{5}$ by (1) with

$\begin{aligned} U_{0}(x, y, z)= & {[\operatorname{erfc} \sqrt{\omega \zeta(x, z)}-\operatorname{erf} \sqrt{\omega \zeta(x-\pi a, z)}][\operatorname{erfc} \sqrt{\omega \zeta(y, z)}-\operatorname{erf} \sqrt{\omega \zeta(y-\pi b, z)}] } \\ & \times \frac{e^{\omega z} \sinh [\omega(\pi-z)]}{\sinh [\omega \pi]} .\end{aligned}$
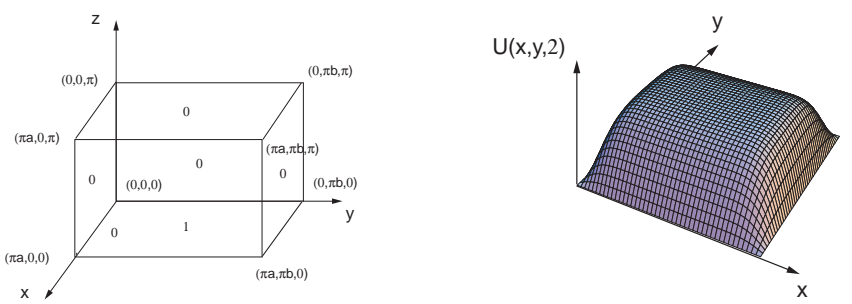

Fig. 5. (a) Domain $\Omega_{5}$ and Dirichlet conditions of problem $\left(P_{5}\right)$ (b) Graph of the first order approximation for the solution of problem $\left(P_{5}\right)$ for $\varepsilon=0.1$.

Then, the first order approximation of the solution of problem $\left(P_{5}\right)$ is a combination of products of error functions. See [7] for further information. 


\section{Conclusions}

It is clear from the above examples that the (complementary) error function plays a fundamental role in the approximation of these problems in $\tilde{\Omega}$ (away from the discontinuities of the boundary conditions) as $\varepsilon \rightarrow 0^{+}$. It seems that the error function shows up as a universal approximant. But this fact is not surprising: the complementary error function

$$
u(x, y ; \tilde{x}, \tilde{y}):=\frac{1}{2} \operatorname{erfc}\left[\frac{1}{\sqrt{2 \varepsilon}} \zeta(x-\widetilde{x}, y-\widetilde{y})\right], \quad(\tilde{x}, \tilde{y}) \quad \text { fixed },
$$

is an exact solution of the $2 \mathrm{D}$ convection-diffusion partial differential equation with constant convection vector $\vec{v}$ and satisfies approximately the Dirichlet data: consider the line defined by the convection vector $\vec{v}$ emanating from a discontinuity point $(\widetilde{x}, \widetilde{y}) \in \partial \Omega$ defined by $\{(x, y) \mid \zeta(x-\widetilde{x}, y-\widetilde{y})=0\}$, then $u(x, y ; \tilde{x}, \tilde{y}) \simeq 0$ at one side of this line and $u(x, y ; \tilde{x}, \tilde{y}) \simeq 1$ at the other side, approximating in this way the boundary condition that only takes the values 0 or 1 . Moreover, this function always lies between the values 0 and 1 and those limiting values are approached rapidly. It exhibits a rapid transition from one value to another when $\vec{r}$ crosses the above mentioned line. A "maximum principle" states that the solution of this problem must have its values between 0 and 1 , so function (2) reproduces this property of the exact solution. Furthermore, the arguments of the complementary error function describe approximately the shape and size of the singular layers as well as their location. The singular parameter $\varepsilon$ controls the incline of the singular layers: the smaller $\varepsilon$ is, the steepest the shape of $U$ is on the singular layer. The size of the transition region (singular layer) is $\mathcal{O}(\sqrt{\varepsilon})$.

The layer structure of the solution of these problems is described by the (complementary) error function or combinations of error functions. From a numerical point of view, these functions can be very useful to design stable numerical methods. For the construction of local grids their arguments give an idea about the mesh size and location of refined meshes. Moreover, in the analysis of any numerical method it is important to derive sharp bounds for the derivatives of the solutions in terms of $\varepsilon$. The derivation of the approximations obtained in $[1,2,3,4,5,6,7]$ may be used to obtain those bounds.

\section{References}

1. J.L. López and E. Pérez Sinusía, Stud. Appl. Math. 113(1), 57-89 (2004).

2. J.L. López and E. Pérez Sinusía, Acta Appl. Math. 82(1), 101-117 (2004).

3. J.L. López and E. Pérez Sinusía, J. Comp. Appl. Math. 181, 1-23 (2005).

4. J.L. López, E. Pérez Sinusía and N.M. Temme, Stud. Appl. Math. 116, 303-319 (2006).

5. J.L. López and E. Pérez Sinusía, P. Roy. Soc. Edinb. A 137A, 93-109 (2007).

6. J.L. López, E. Pérez Sinusía and N.M. Temme, J. Math. Anal. Appl. 328(2), 931-945 (2007).

7. J.L. López and E. Pérez Sinusía, IMA. J. Appl. Math. to appear (2008) 